\title{
Three dimensional (3D) Microstructure-Based Finite Element Modeling of Al-SiC Nanolaminates Using Focused Ion Beam (FIB) Tomography
}

\author{
C.R. Mayer ${ }^{1}$, J. Molina-Aladareguia ${ }^{2}$ and N. Chawla ${ }^{1} *$ \\ ${ }^{1}$ Materials Science and Engineering \\ Arizona State University \\ Tempe, AZ 85287-6106 \\ 2 IMDEA Materials Institute \\ c/Eric Kandel 2, Getafe, Madrid \\ 28906, Spain \\ Submitted to: \\ Materials Characterization
}

*Corresponding author: nchawla@asu.edu, 480-965-2402,

501 E. Tyler Mall, ECG 303

Arizona State University

Tempe, AZ 85287-6106 


\begin{abstract}
$\mathrm{Al}-\mathrm{SiC}$ nanolaminate composites show promise as high performance coating materials due to their combination of strength and toughness. Although a significant amount of modeling effort has been focused on materials with an idealized flat nanostructure, experimentally these materials exhibit complex undulating layer geometries. This work utilizes FIB tomography to characterize this nanostructure in 3D and finite element modeling to determine the effect that this complex structure has on the mechanical behavior of these materials. A sufficiently large volume was characterized such that a $1 \times 2 \mu \mathrm{m}$ micropillar could be generated from the dataset and compared directly to experimental results. The mechanical response from this nanostructure was then compared to pillar models using simplified structures with perfectly flat layers, layers with sinusoidal waviness, and layers with arc segment waviness. The arc segment based layer geometry showed the best agreement with the experimentally determined structure, indicating it would be the most appropriate geometry for future modeling efforts.
\end{abstract}

Keywords: Micropillar compression, multilayer, waviness, nanostructure, FEA 


\section{List of Figures}

Figure 1: SEM image of nanolaminate nanostructure, showing significant waviness in the deposited layers.

Figure 2: Outline of the image processing steps used to segment the $\mathrm{Al}$ and $\mathrm{SiC}$ phases from the raw images.

Figure 3: Pillar models used with flat, cosine, arc segment, and FIB derived nanostructures (respectively from left to right). Al layers are depicted in green while $\mathrm{SiC}$ layers are depicted in gray.

Figure 4: Comparison of experimental pillar compression response [12] to the modeled response using the FIB tomography derived nanostructure.

Figure 5: Modeled stress strain response of the flat nanostructure pillar. Von Mises stress contours correspond to the points indicated on the curve.

Figure 6: Modeled stress strain response of the cosine nanostructure pillar. Von Mises stress contours correspond to the points indicated on the curve.

Figure 7: Modeled stress strain response of the arc segment nanostructure pillar. Von Mises stress contours correspond to the points indicated on the curve.

Figure 8: Modeled stress strain response of the FIB derived nanostructure pillar. Von Mises stress contours correspond to the points indicated on the curve.

Figure 9: Comparison of modeled responses for all four layer geometries showing that the arc structure most closely approximates the FIB derived structure.

Figure 10: Distribution and cumulative distribution of element stresses in the loading direction for each of the structures at 0.005 strain. The arc segment structure fits the results from the FIB 
structure the most accurately, indicating it is the most suitable for capturing how the stresses are being localized. 


\section{Introduction}

Laminated composite structures have long been used to tailor the properties of materials for specific applications by varying the composition, scale, and volume fraction of the individual

phases used. By decreasing the thickness of the layers to the nanoscale, very interesting mechanical properties can be obtained, such as high strength, toughness, and wear resistance [16]. Additionally, these nanolaminate composites have been shown to exhibit unique functional properties such as biocompatibility [7], and enhanced optical properties [8].

An ever growing body of literature has focused on these materials. In an effort to understand the underlying deformation mechanisms, several papers have dealt with modeling approaches over a range of length scales to better understand different aspects of the mechanical behavior. These methods have included molecular dynamics (MD) simulations $[9,10]$ which allows detailed investigation of interfaces and dislocations but with very limited size and time scales, molecular statistics (MS) simulations [11] which provides similar information as MD simulations with reduced computation time, dislocation dynamics (DD) simulations [12] which can be used to determine the transitions in dislocation behavior seen in multilayers as a function of layer thickness, and finite element (FE) modeling [13-15] which can determine how the stresses and strains are distributed within heterogeneous structures even under complex loading conditions such as indentation. 
The vast majority of this literature assumes a perfectly flat layer structure $[6,11,14,15]$, which is not representative of the actual nanostructure, as seen in Figure 1. The waviness in the layers is a byproduct of the deposition process and has been observed experimentally in a number of multilayer systems [16-19]. This type of columnar growth structure occurs because any small amount of roughness is exaggerated upon application of another layer, eventually leading to shading and porosity [20]. Experimentally this difference in the nanostructure results in significant changes in the observed deformation behavior. This is especially apparent in recent work using micropillar compression [21], where waviness in the layers was shown to both allow buckling of the layers to occur at much lower stresses and also hinder shear deformation within individual layers. Additionally under nanoindentation loading, the increased waviness was shown make the measured moduli more isotropic [18] and the stresses more localized [18, 19]. Some more recent studies have begun implementing non-ideal structures to account for this, for example layers with sinusoidal waviness [13, 18, 21, 22] or arc segment waviness [19].

Finite element (FE) modeling using experimentally obtained microstructures can provide more accurate results as well as meaningful insights into the deformation behavior compared to simple model systems [23-26]. A variety of experimental methods are available to characterize materials in three dimensions (3D). These techniques range from relatively coarse techniques such as optical sectioning microscopy [26], to x-ray computed tomography with sub-micrometer resolution [27], and to atom probe tomography with individual atom location attainable [28]. The length scales of interest in this study of nanolaminate materials (requiring nanometer scale resolution over tens of cubic micrometers of volume) makes focused ion beam (FIB) tomography the most appropriate compromise between the required resolution and volume of material which 
needs to be characterized. This technique utilizes a dual beam FIB/SEM to image the structure with a high resolution SEM while sequentially removing thin slices of material (typically on the order of tens of nanometers) with the FIB [29]. The depth resolution of this technique is determined by the slice thickness, which in theory is limited by the resolution of the ion beam and can therefore achieve resolutions at the nanometer scale, although, in the interest of efficiency, larger slices on the order of tens of nanometer are often removed to reduce the time required. Additionally, with FIB tomography volumes as large as tens of cubic micrometers of material can be characterized. This volume is large enough to allow a $1 \mu \mathrm{m}$ diameter by $2 \mu \mathrm{m}$ height pillar to be cropped from the reconstructed volume and used in simulations, allowing direct comparison to experimental compression experiments carried out in previous work [21].

For the case of these nanolaminate materials, the amplitude and wavelength of the waviness varies from sample to sample depending on the exact processing parameters, layer thicknesses, and materials used, making it impractical to experimentally determine the microstructure in $3 \mathrm{D}$ for every sample. Thus, the aim of this work is to determine how appropriate perfectly flat, sinusoidal, and arc segment nanostructures are for modeling the deformation of these laminate structures. By comparing the simulated mechanical responses of each of these simplified structures to the simulated response from experimentally determined structure obtained using FIB tomography, as well as experimental pillar compression results from previous work [21], we are able to determine which idealized structure provides the most accurate approximation of both the constitutive stress-strain relations as well as the localization of deformation within each structure. 


\section{Materials and Methods}

The metal-ceramic nanolaminate composites were fabricated using an automated magnetron sputtering procedure, the details of which are given elsewhere $[9,10]$. The samples used in this study consisted of alternating layers of $\mathrm{Al}$ and $\mathrm{SiC}$, each having an individual layer thickness of approximately $50 \mathrm{~nm}$. These layers were deposited sequentially until a total multilayer thickness of approximately $15 \mu \mathrm{m}$ was obtained (approximately 300 layers).

FIB tomography was carried out in a dual beam FIB/SEM (Nova200, FEI, Hillsboro, OR). Cross-sections approximately $5 \mu \mathrm{m}$ x $5 \mu \mathrm{m}$ in size were imaged following each milling step. Slices of $80 \mathrm{~nm}$ each were removed in each step using the FIB. Ion beam images normal to the sample surface were used to measure the distance from the cross section to a reference fiduciary mark, thereby providing an accurate measure of the slice thickness. The cross section cut using the FIB was then imaged at 52 degrees relative to the surface normal. The images were scaled along the cross section height in order to compensate for this imaging angle. A total of 23 slices were imaged for a total depth of $1.8 \mu \mathrm{m}$. These slices were then manually aligned using the Avizo Fire (VSG, Burlington, MA) software. A volume of $3.2 \mu \mathrm{m}$ by $3.5 \mu \mathrm{m}$ by $1.8 \mu \mathrm{m}$ was cropped from these aligned slices in order to both remove the misaligned edges as well as provide a volume of material with maximum contrast.

Before the 3D volume characterized using FIB tomography could be utilized in a FE model, the $\mathrm{Al}$ and $\mathrm{SiC}$ phases needed to be segmented in each of the 2D slices. As the contrast differences in the raw images were not adequate for grayscale thresholding immediately, a variety of image 
manipulations were implemented (Image J, Bethesda, MD) in order to improve the image quality, as shown in Figure 2. First, in order to remove the background contrast variations, a Gaussian blur was applied to the image and then this blurred image was subtracted from the original. The radius of this Gaussian blur was adjusted to the minimum size where any feature of interest is not visible in the blurred image, in this case $50 \mathrm{~nm}$. Since the features of interest in this case were the horizontal layered structure, a mask was applied to the Fourier transform of the image in order to remove much of the noise without affecting the horizontal features. This process provided images which were able to be segmented using standard grayscale thresholding. Due to contrast differences, areas immediately surrounding pores in the material required manual adjustment to ensure layer continuity. As the slice thickness was much larger than the $x$ and y pixel size, the image stack resulted in non-cubic voxels. In order to obtain uniform cubic voxels, the 3D volume was resampled with layer positions interpolated linearly between the slices. Avizo Fire was then used to generate a 3D solid tetrahedral mesh of the structure.

Three simplified nanostructure geometries, with layers consisting of either flat, cosine or arc sections, were developed in order to approximate the experimental structure. Using a single cross sectional image, the average amplitude and wavelength of the undulations were measured to be $50 \mathrm{~nm}$ and $475 \mathrm{~nm}$, respectively. Equivalent amplitude and wavelength values were used while generating the cosine and arc section based geometries.

In order to mimic experimental pillar compression studies, $1 \mu \mathrm{m}$ diameter by $2 \mu \mathrm{m}$ height pillars were cropped from each of the geometries as seen in Figure 3. A $2^{\circ}$ taper was also incorporated 
in the pillar models, as seen experimentally [21]. In each of the models the layers were oriented parallel to the loading axis, as this orientation showed the strongest dependence on layer waviness, as per previous studies [18, 21].

The deformation of the micropillars was simulated by FE modeling using the commercial software Abaqus (Abaqus, v. 6.12, Dassault Systems Simulia Corp., Providence, RI). All the pillar models were meshed using 4-node tetrahedral elements (C3D4). To determine the number of elements required for mesh convergence, the cosine geometry mesh was iteratively refined in $\sim 100,000$ element steps until deviations in the peak load were determined to be less than $1 \%$ from the previous iteration, which occurred at $\sim 600,000$ elements. This element count was used for the cos, arc and flat geometries, while the FIB derived mesh generated from the Avizo Fire program contained $\sim 1,000,000$ elements. The bottom surface of the pillar was completely constrained while the top surface was constrained in the $\mathrm{x}$ and $\mathrm{z}$ dimensions and displaced as a function of time in the y dimension in order to induce the deformation. Displacement was carried out at a rate of $2 \mathrm{~nm} / \mathrm{s}$ to a maximum of $200 \mathrm{~nm}$, corresponding to an initial strain rate of $0.001 \mathrm{~s}^{-1}$ and a maximum strain of 0.1 . During loading, this set of constraints is analogous to displacement controlled micropillar compression with a rigid indenter and high contact friction between the indenter and pillar top. A deformable base material was excluded from all models because this additional source of compliance has already been accounted for using the Sneddon correction [6] in the experimental results being used for comparison [21].

The constitutive behavior used for the $\mathrm{Al}$ and $\mathrm{SiC}$ phases has been utilized in previous studies $[15,30]$ and was approximated from the results of indentations made on $1 \mu \mathrm{m}$ thick monolithic 
films of each phase, using the same deposition parameters. For the aluminum phase, the elastic modulus and Poisson's ratio were $59 \mathrm{GPa}$ and 0.33 , respectively, with an initial yield point of $200 \mathrm{MPa}$. A piecewise hardening behavior following yield was used with linear hardening between (stress, plastic strain) points of (200 MPa, 0), (300 MPa, 0.5), and (400 MPa, 3), followed by perfectly plastic behavior. For the SiC phase, the elastic modulus and Poisson's ratio was $277 \mathrm{GPa}$ and 0.18 , respectively. A yield point of $8770 \mathrm{MPa}$ was chosen, followed by perfect plasticity. Although the brittle $\mathrm{SiC}$ is not expected to undergo significant plasticity experimentally, unrealistically high stresses occur using purely elastic behavior due to the lack of fracture incorporated into the models. Therefore, allowing perfectly plastic deformation after a high yield (essentially fracture) point estimated from the indentation hardness allows for a closer approximation of the actual behavior. The boundaries between the $\mathrm{Al}$ and $\mathrm{SiC}$ phases are modeled as perfect interfaces, an assumption which has been shown to provide strong agreement with experimental results at room temperature [14].

\section{Results and Discussion}

A comparison of experimental pillar compression curves with the simulated result from the FIB determined microstructure is shown in Figure 4. The model shows very good agreement with the experimental data, with the maximum stress in the model falling within the range reported for the experimental pillars. One notable difference between the model and the experimental pillars is that the measured modulus in the experimental stress strain curves is much lower than the modeled behavior. This lower apparent modulus has also been seen in previous studies when comparing the modulus calculated using the stress strain curve and the continuous stiffness 
measurement (CSM) based modulus [6]. The root of this difference is extraneous contributions to the compliance. These can include underestimates of the Sneddon correction to the sink in effect, additional compliance in the instrument load frame, slight misalignments or surface asperities on the pillar top causing imperfect contact $[6,31]$. Due to the electrostatic actuation of the indenter tip, the experimental pillar compression results are inherently load controlled, resulting in large displacement jumps at fracture events or plastic instabilities. Therefore, for the experimental data, failure is defined at the first instance of a negative hardening rate and denoted by a downward arrow.

Stress strain curves and corresponding frames showing the Von Mises stress contours for the FIB, flat, cosine and arc segment structures are shown in Figure 5 - 8, respectively. Videos of the deformation are provided in the supplemental material. The fully deformed pillars using the experimental and arc segment structures (Figure 7 and Figure 8 (c)) show significant buckling of the layers in a single localized region. This is also observed experimentally [21], although the lateral constraint imposed on the top surface of the modeled pillars likely reduces this effect. Directly comparing the stress strain curves from each of the models indicates that, based on the overall mechanical response, the arc segment based nanostructure is the best approximation of the experimentally determined structure, as seen in Figure 9.

In addition to the overall mechanical response quantified by the stress strain curves, it is also important to determine which model best approximates the localization of stresses in the structure. This is especially relevant to fracture processes, where the high local stresses can drive flaw propagation. In order to quantify this localization, the distribution of stresses along the loading axis (S22) was quantified at a strain of 0.005 , which is within the elastic regime. For 
each of the models, the distribution of the stresses in the $\mathrm{SiC}$ elements was calculated as shown in Figure 10. In order to quantify how well the localization in the FIB determined structure is reproduced by each of the simplified geometries, the $\mathrm{R}^{2}$ parameter was used to determine the goodness of fit. The $\mathrm{R}^{2}$ values for the flat, cosine, and arc segment structures were determined to be $-1.67,0.86$, and 0.95 , respectively. This indicates that the arc segment model approximates the localization of stresses the most accurately as well.

It is also interesting to note where the stresses are being localized within the structure. In each of the structures incorporating waviness, the stresses are being localized in the areas with the highest curvature. In the cosine structure, this is at both the peaks and troughs of the waviness, in the arc segment and FIB determined structure, this is at only the troughs of the waviness. In the real nanolaminate structure, this localization is especially detrimental because of the porosity. Due to shading effects in the deposition process the troughs (i.e. columnar boundaries) also have a high propensity for void formation (as seen in Figure 1). The combination of inherently weak areas due to voids along with stresses which can be larger by a factor of two than the far field stress can lead to early failure of the material.

These weak spots in the structure can have significant effects on the mechanical properties of the laminate. For one, they can act as preexisting flaws which would explain the strong Weibull size effect observed in pillar compression [21]. The low strength of the columnar boundaries also plays a role in the fracture toughness of these materials. At small layer thicknesses $(\sim 10 \mathrm{~nm})$ where plasticity in the highly constrained $\mathrm{Al}$ layers is low, the fracture surfaces indicate that the fracture path is dominated by propagation through columnar boundaries [32]. 


\section{Conclusions}

This work has provided insight into the role the nanostructure plays in the deformation behavior of Al-SiC nanolaminate composites allowing the following conclusions to be drawn;

- The waviness of the structure makes a significant contribution to the mechanical response of these materials and needs to be accounted for in order to obtain even qualitative agreement with experiment.

- Close agreement between experimental pillar compression and the modeled response of the FIB determined nanostructure was observed in the stress vs plastic strain behavior.

- Of the simplified structures used for modeling, the structure consisting of arc segments provided the stress strain response closest to the one obtained using the FIB tomography determined nanostructure

- The arc segment based nanostructure was also able to closely reproduce the distribution of $\mathrm{SiC}$ element stresses seen in the FIB tomography determined nanostructure, indicating that the stresses are localized in a similar fashion.

- The two above conclusions indicate that when quantitative modeling results are required, a layer geometry based on arc segments should be utilized.

\section{Acknowledgements}

The authors would like to acknowledge the National Science Foundation Materials World Network (Contract DMR-1209928, Dr. Lynnette Madsen, Program Manager) for financial 
support of this research and the LeRoy Erying Center for Solid State Science for the use of their microscopy facilities.

\section{References}

[1] M. Shinn, L. Hultman, and S. Barnett, "Growth, structure, and microhardness of epitaxial TiN/NbN superlattices,” Journal of Materials Research, vol. 7, pp. 901-911, 1992.

[2] U. Wiklund, P. Hedenqvist, and S. Hogmark, "Multilayer cracking resistance in bending," Surface and Coatings Technology, vol. 97, no. 1-3, pp. 773 - 778, 1997.

[3] N. Chawla, D. Singh, Y.-L. Shen, G. Tang, and K. Chawla, "Indentation mechanics and fracture behavior of metal/ceramic nanolaminate composites," Journal of Materials Science, vol. 43, pp. 4383-4390, 2008.

[4] D. Singh and N. Chawla, "Scratch resistance of Al/SiC metal/ceramic nanolaminates," Journal of Materials Research, vol. 27, pp. 278-283, 2012.

[5] N. A. Mara, D. Bhattacharyya, P. Dickerson, R. G. Hoagland, and A. Misra, "Deformability of ultrahigh strength $5 \mathrm{~nm} \mathrm{Cu}-\mathrm{Nb}$ nanolayered composites," Applied Physics Letters, vol. 92, no. 23, pp. 1-3, 2008.

[6] D. Singh, N. Chawla, G. Tang, and Y.-L. Shen, "Micropillar compression of Al/SiC nanolaminates," Acta Materialia, vol. 58, no. 20, pp. 6628-6636, 2010.

[7] W. Li, B. Kabius, and O. Auciello, "Science and technology of biocompatible thin films for implantable biomedical devices," in Engineering in Medicine and Biology Society (EMBC), 2010 Annual International Conference of the IEEE, 2010, pp. 6237-6242. 
[8] G. S. Hickey, S.-S. Lih, and T. W. Barbee, Jr., "Development of nanolaminate thin-shell mirrors," Proc. SPIE, vol. 4849, pp. 63-76, 2002.

[9] Y. Wang, J. Li, A. V. Hamza, and T. W. Barbee, "Ductile crystalline-amorphous nanolaminates," Proceedings of the National Academy of Sciences, vol. 104, no. 27, pp. 1115511160, 2007.

[10] R. Zhang, T. Germann, J. Wang, X.-Y. Liu, and I. Beyerlein, "Role of interface structure on the plastic response of $\mathrm{Cu} / \mathrm{Nb}$ nanolaminates under shock compression: Non-equilibrium molecular dynamics simulations," Scripta Materialia, vol. 68, no. 2, pp. 114 - 117, 2013. [11] M. Liu, J. Huang, Y. Fong, S. Ju, X. Du, H. Pei, and T. Nieh, “Assessing the interfacial strength of an amorphous-crystalline interface," Acta Materialia, vol. 61, no. 9, pp. 3304 - 3313, 2013.

[12] N. M. Ghoniem and X. Han, "Dislocation motion in anisotropic multilayer materials," Philosophical Magazine, vol. 85, no. 24, pp. 2809-2830, 2005.

[13] R. D. Jamison and Y.-L. Shen, "Indentation and overall compression behavior of multilayered thin-film composites: Effect of undulating layer geometry," Journal of Composite Materials, 2015.

[14] S. Lotfian, M. Rodriguez, K. Yazzie, N. Chawla, J. Llorca, and J. Molina-Aldareguia, "High temperature micropillar compression of Al/SiC nanolaminates," Acta Materialia, vol. 61, no. 12 , pp. $4439-4451,2013$.

[15] G. Tang, Y.-L. Shen, D. Singh, and N. Chawla, "Indentation behavior of metal-ceramic multilayers at the nanoscale: Numerical analysis and experimental verification," Acta Materialia, vol. 58, no. 6, pp. $2033-2044,2010$. 
[16] Y. Chen, Y. Liu, C. Sun, K. Yu, M. Song, H. Wang, and X. Zhang, "Microstructure and strengthening mechanisms in Cu/Fe multilayers," Acta Materialia, vol. 60, no. 18, pp. 6312$6321,2012$.

[17] G. Zhang, T. Fan, T. Wang, and H. Chen, "Microstructure, mechanical and tribological behavior of MoNx/SiNx multilayer coatings prepared by magnetron sputtering," Applied Surface Science, vol. 274, no. 0, pp. $231-236,2013$.

[18] C. Mayer, L. Yang, S. Singh, H. Xie, Y.-L. Shen, J. Llorca, J. Molina-Aldareguia, and N. Chawla, "Orientation dependence of indentation behavior in Al-SiC nanolaminate composites," Materials Letters, vol. 168, pp. 129 - 133, 2016.

[19] N. Verma and V. Jayaram, "Role of interface curvature on stress distribution under indentation for ZrN/Zr multilayer coating," Thin Solid Films, vol. 571, Part 2, no. 0, pp. 283 289, 2014, multilayers 2013.

[20] G. S. Bales and A. Zangwill, "Macroscopic model for columnar growth of amorphous films by sputter deposition," Journal of Vacuum Science \& Technology A, vol. 9, no. 1, pp. 145$149,1991$.

[21] C. Mayer, L. Yang, S. Singh, J. Llorca, J. Molina-Aldareguia, Y. Shen, and N. Chawla, "Anisotropy, size, and aspect ratio effects on micropillar compression of Al-SiC nanolaminate composites," Acta Materialia, vol. 114, pp. 25-32, 2016.

[22] R. Jamison and Y.-L. Shen, "Indentation behavior of multilayered thin films: Effects of layer undulation," Thin Solid Films, vol. 570, Part B, pp. 235 - 242, 2014, 2013 International Thin Films Conference.

[23] N. Chawla, B. Patel, M. Koopman, K. Chawla, R. Saha, B. Patterson, E. Fuller, and S. Langer, "Microstructure-based simulation of thermomechanical behavior of composite 
materials by object-oriented finite element analysis," Materials Characterization, vol. 49, no. 5, pp. $395-407,2003$.

[24] M. S. Qidwai, A. C. Lewis, and A. B. Geltmacher, "Using image-based computational modeling to study microstructure-yield correlations in metals," Acta Materialia, vol. 57, no. 14, pp. $4233-4247,2009$.

[25] N. Chawla, R. Sidhu, and V. Ganesh, "Three-dimensional visualization and microstructure-based modeling of deformation in particle-reinforced composites," Acta Materialia, vol. 54, no. 6, pp. 1541 - 1548, 2006.

[26] R. Sidhu and N. Chawla, "Three-dimensional (3D) visualization and microstructurebased modeling of deformation in a Sn-rich solder," Scripta Materialia, vol. 54, no. 9, pp. 1627 1631, 2006.

[27] S. S. Singh, J. J. Williams, T. J. Stannard, X. Xiao, F. D. Carlo, and N. Chawla, "Measurement of localized corrosion rates at inclusion particles in AA7075 by in situ three dimensional (3d) x-ray synchrotron tomography," Corrosion Science, vol. 104, pp. 330 - 335, 2016.

[28] J. Weber, J. Barthel, F. Brandt, M. Klinkenberg, U. Breuer, M. Kruth, and D. Bosbach, "Nano-structural features of barite crystals observed by electron microscopy and atom probe tomography," Chemical Geology, vol. 424, pp. 51 - 59, 2016.

[29] D. Singh, N. Chawla, and Y.-L. Shen, "Focused ion beam (FIB) tomography of nanoindentation damage in nanoscale metal/ceramic multilayers," Mater Char, vol. 61, no. 4, pp. 481-488, 2010. 
[30] G. Tang, Y.-L. Shen, D. Singh, and N. Chawla, "Indentation behavior of metal-ceramic multilayers at the nanoscale: Numerical analysis and experimental verification," Acta Materialia, vol. 58, no. 6, pp. 2033-2044, 2010.

[31] J. R. Greer, W. C. Oliver, and W. D. Nix, "Size dependence of mechanical properties of gold at the micron scale in the absence of strain gradients," Acta Materialia, vol. 53, no. 6, pp. 1821-1830, 2005.

[32] C. Mayer, L. Yang, V. Carollo, J. Llorca, J. Molina-Aldareguia, and N. Chawla, "Microcantilever bending experiments in Al-SiC nanolaminate composites: Characterization of tension-compression asymmetry and fracture toughness as a function of crack orientation and layer thickness,” 2016, Unpublished Manuscript. 


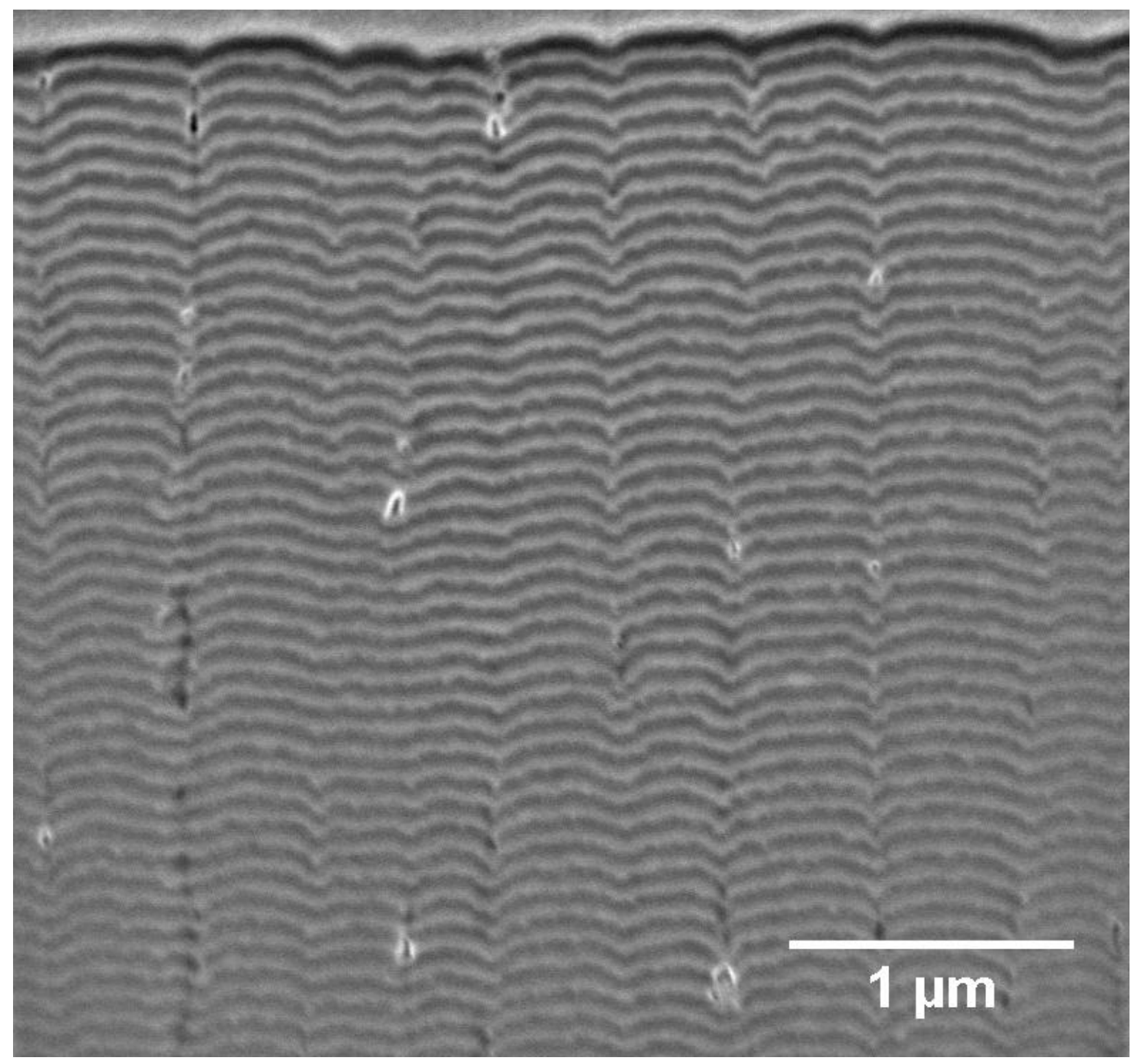




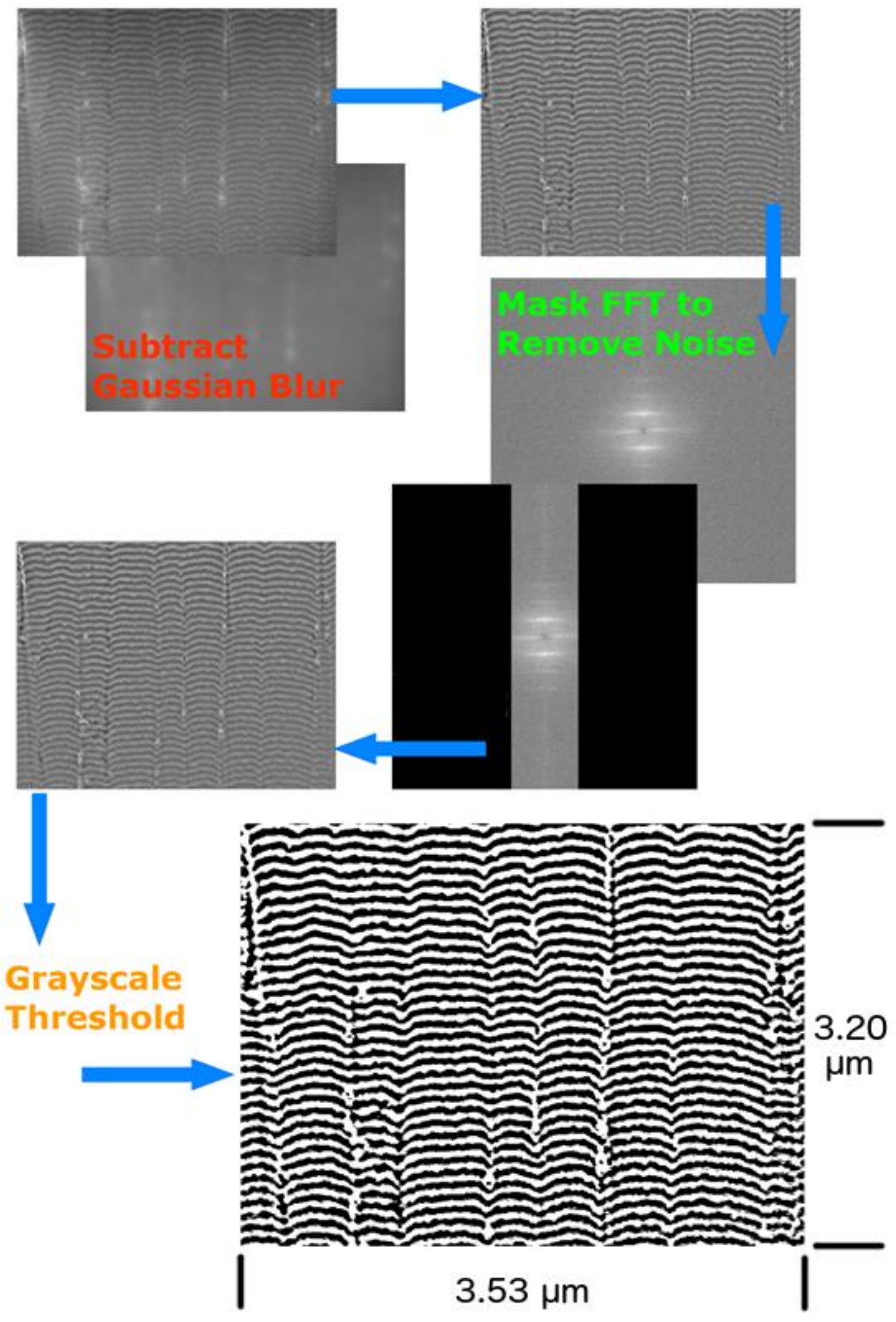



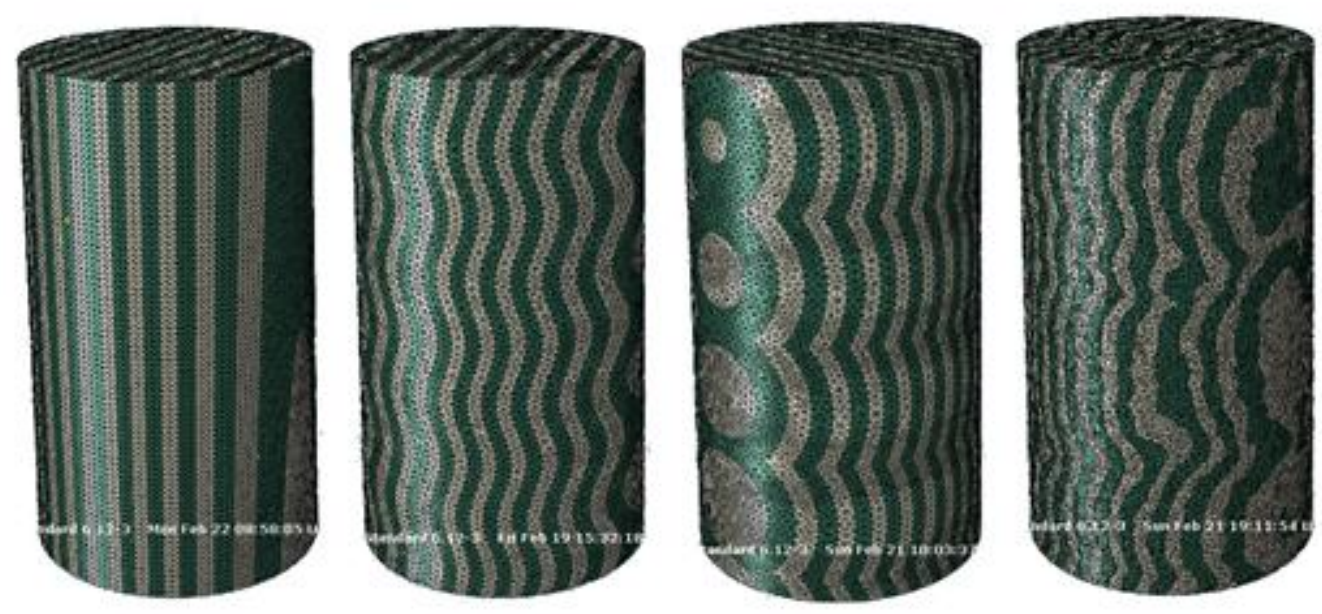


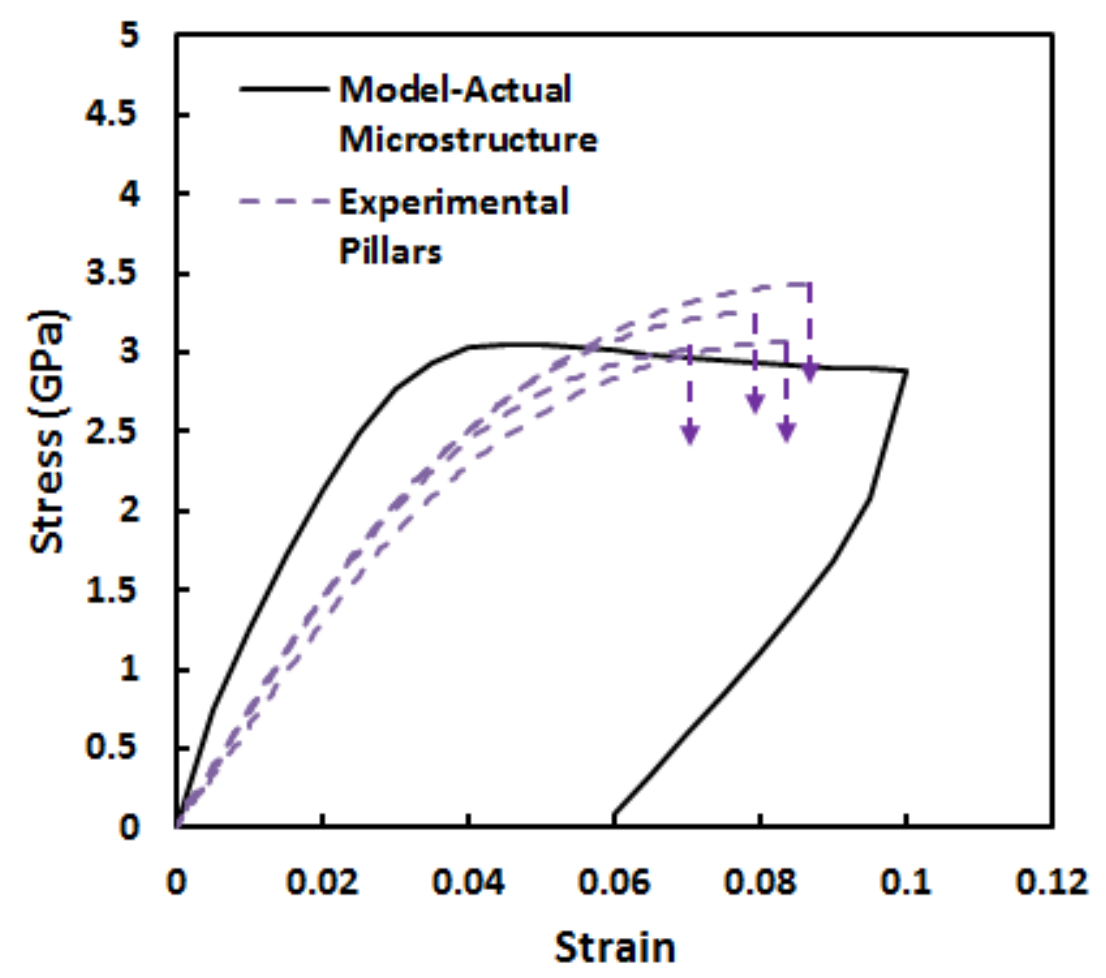




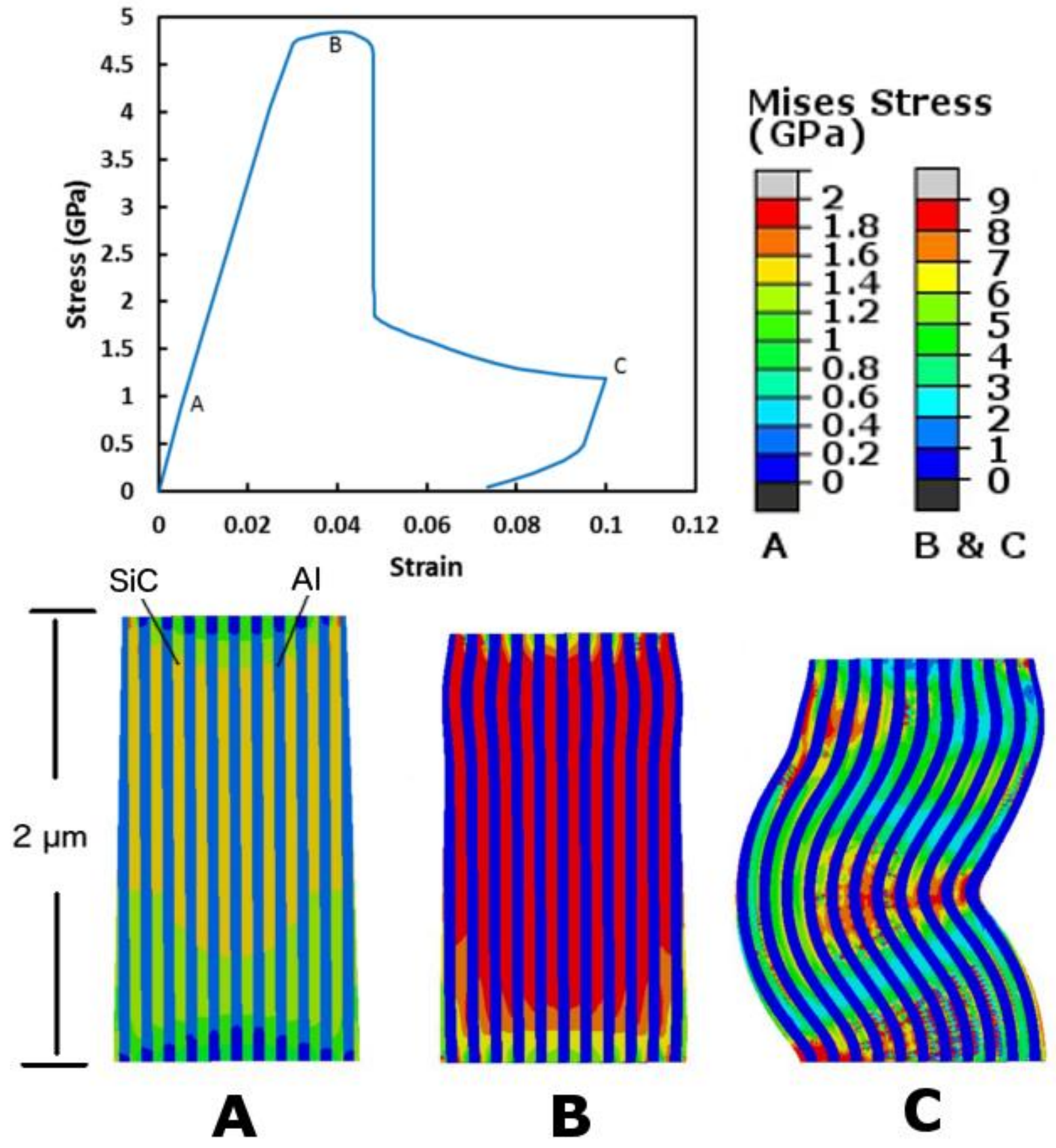




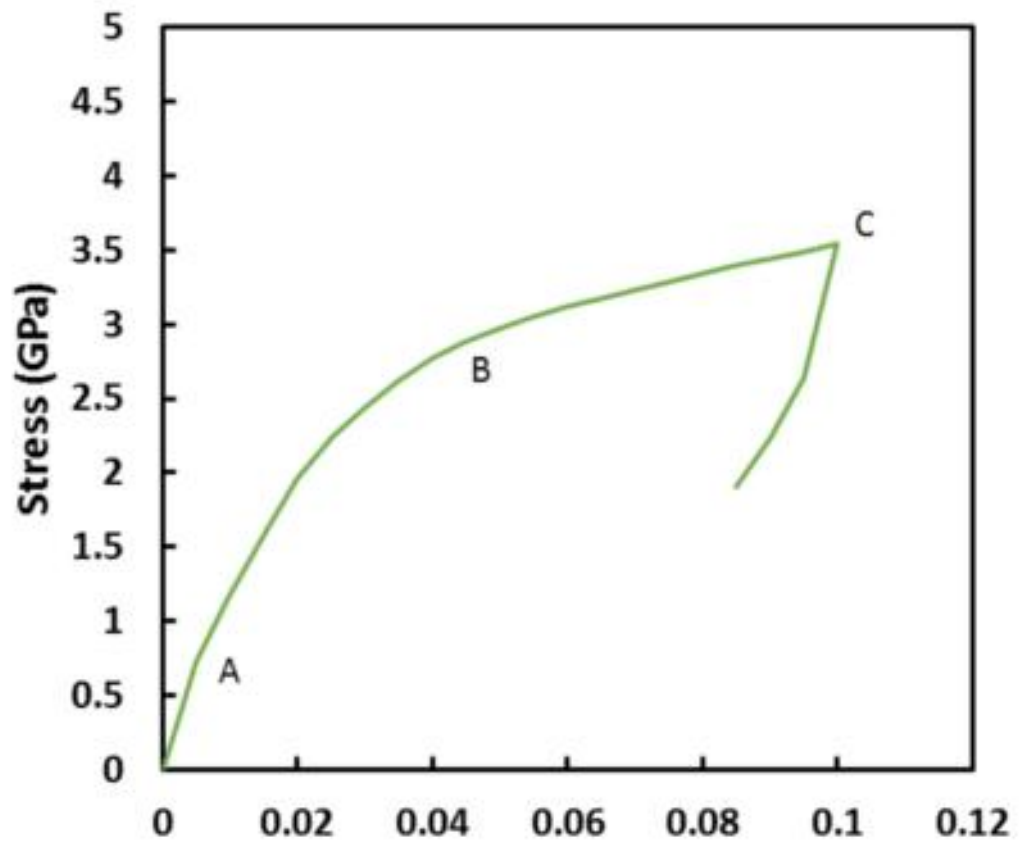

Mises Stress (GPa)
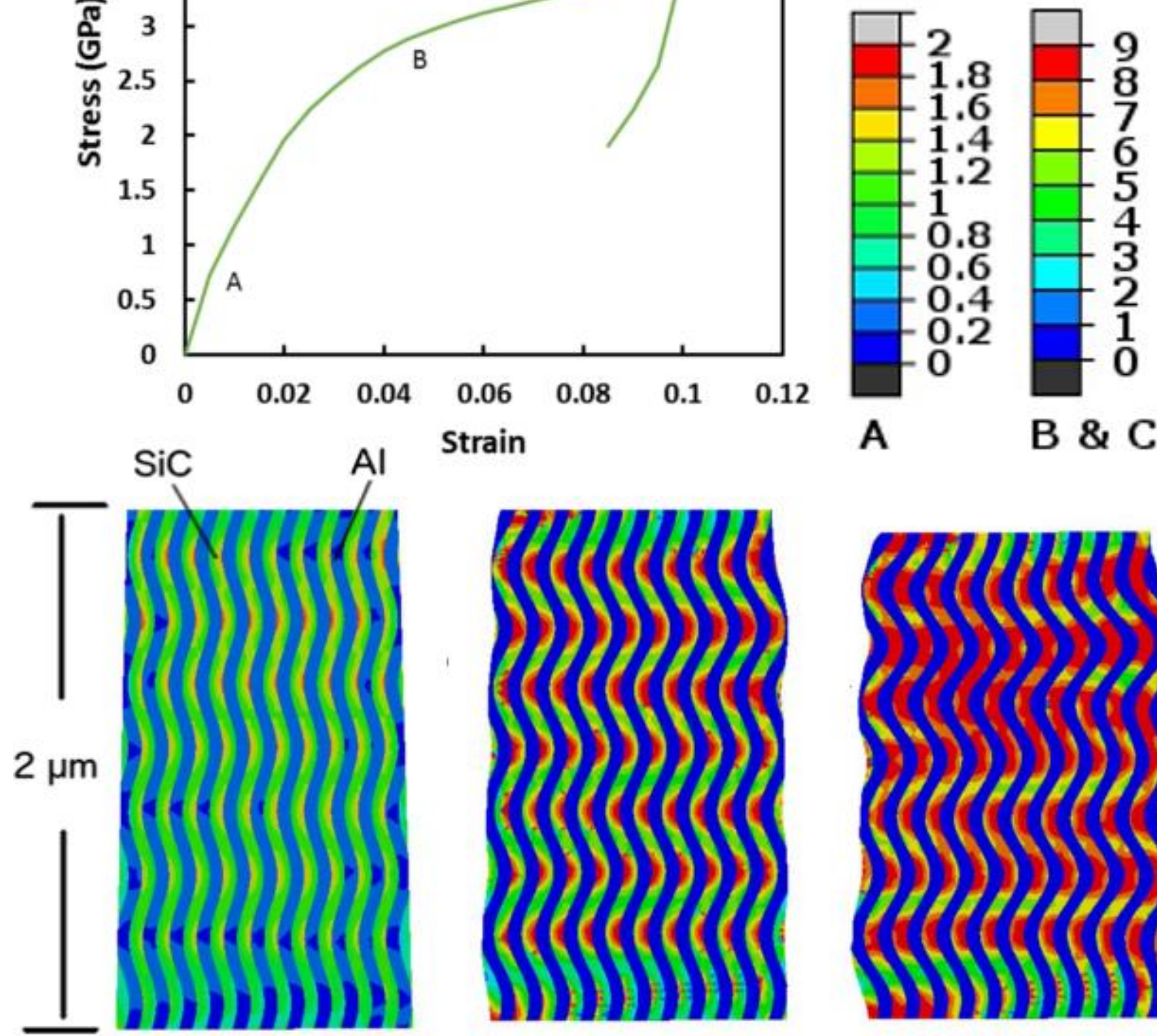
Strain

A
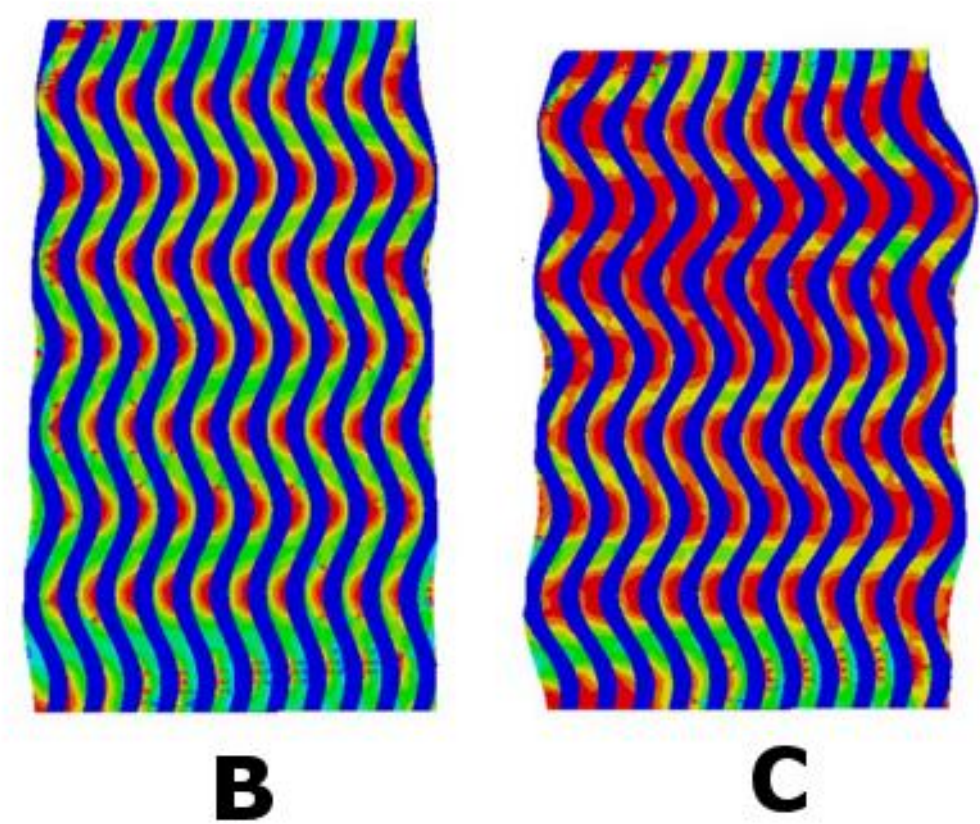


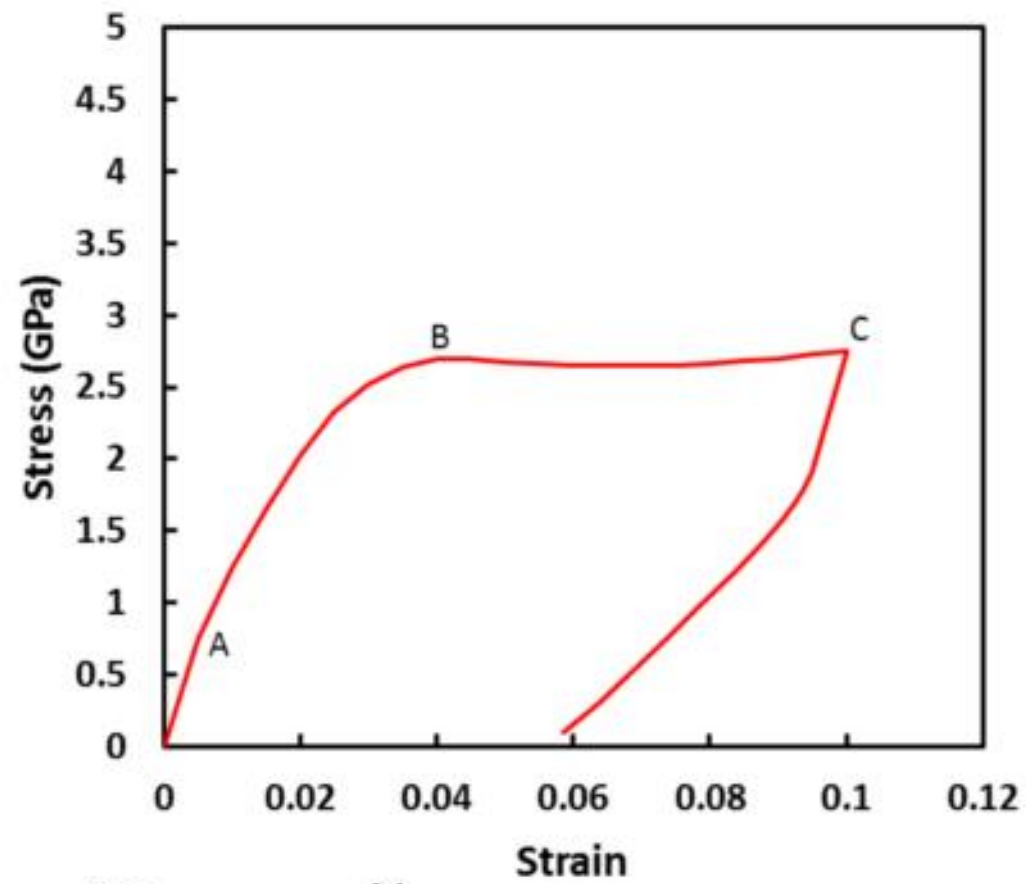

Mises Stress (GPa)
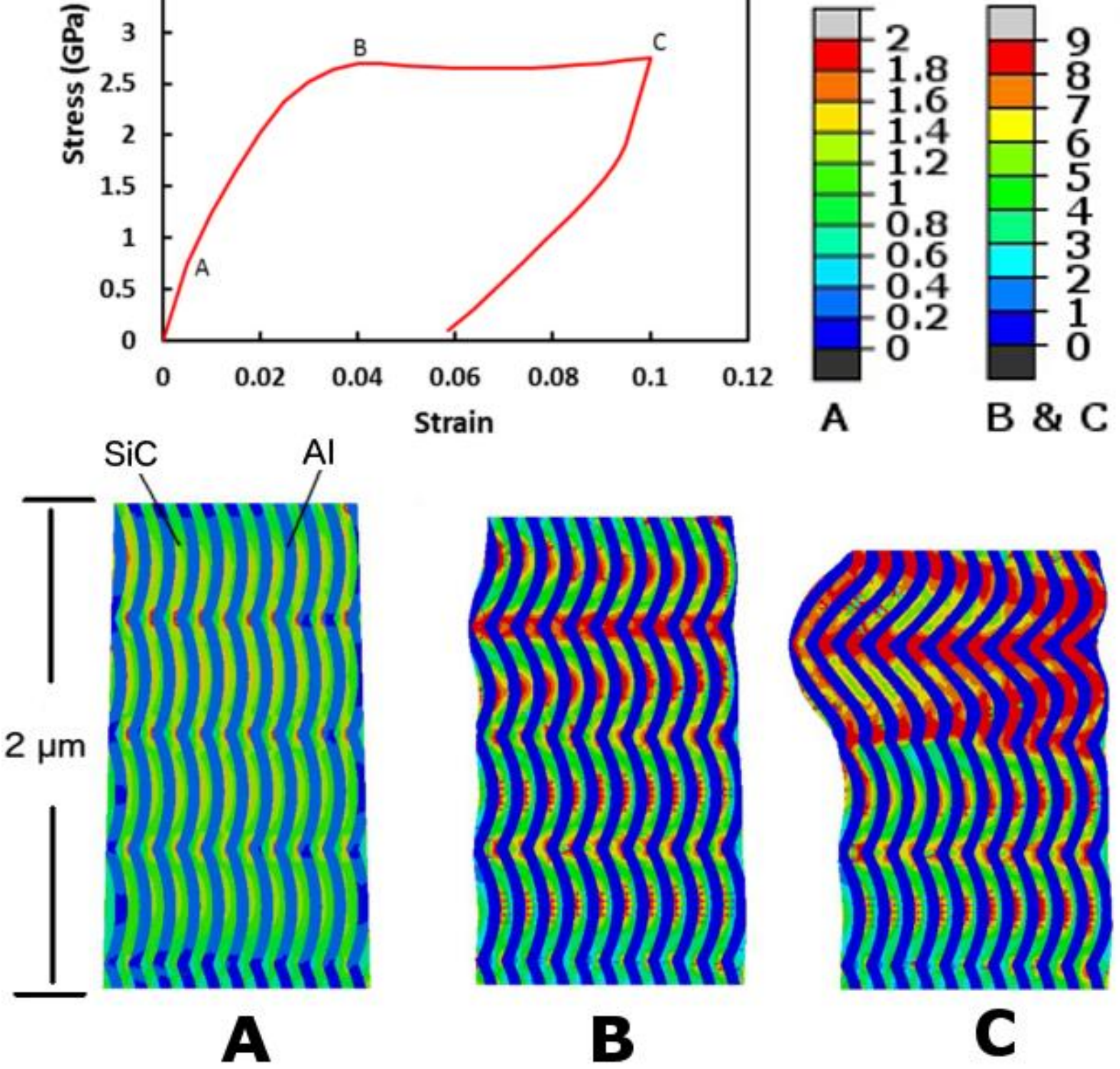


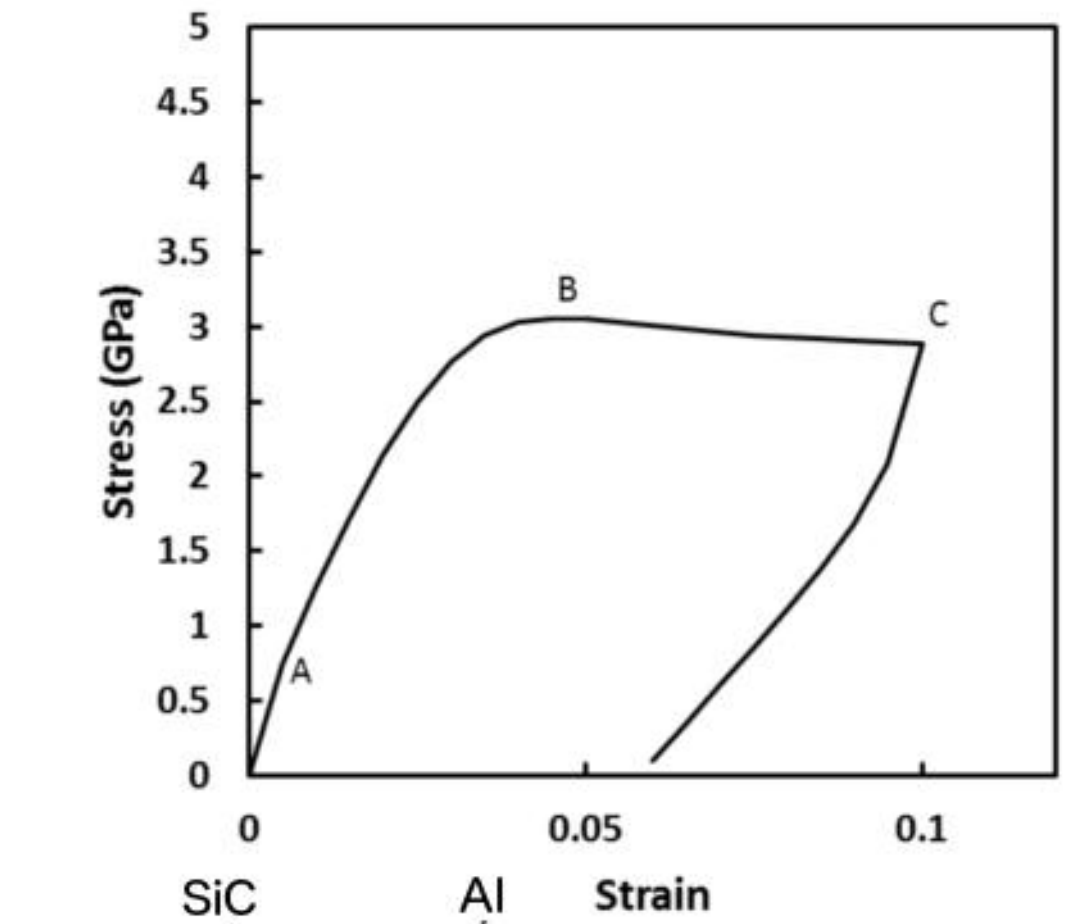

Mises Stress (GPa)

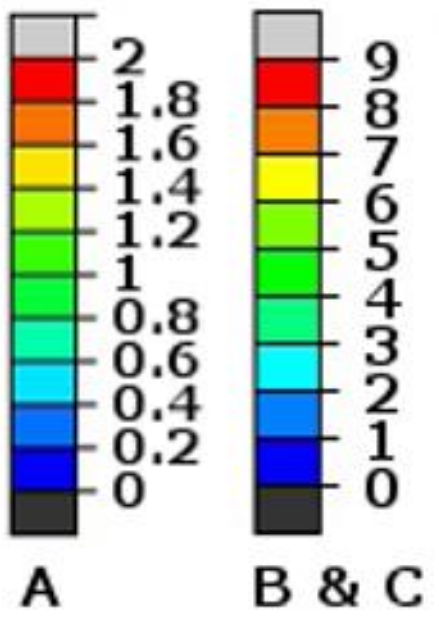

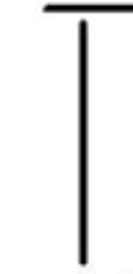

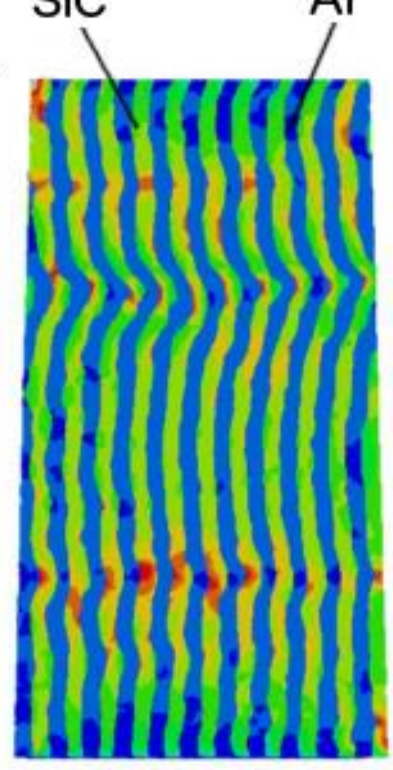

A
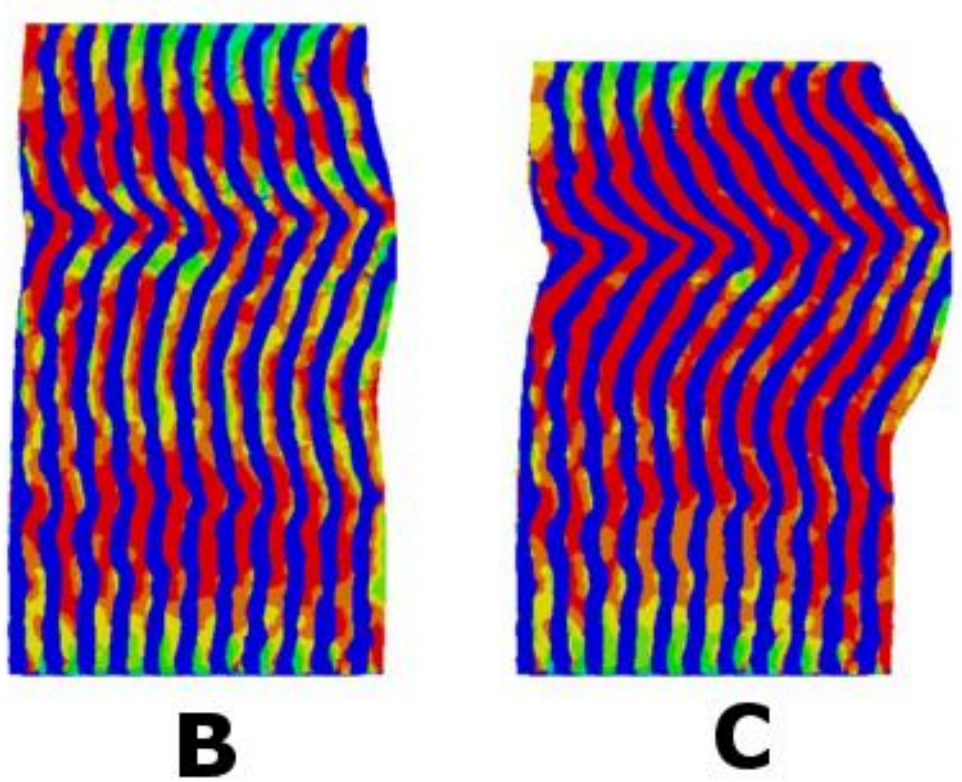


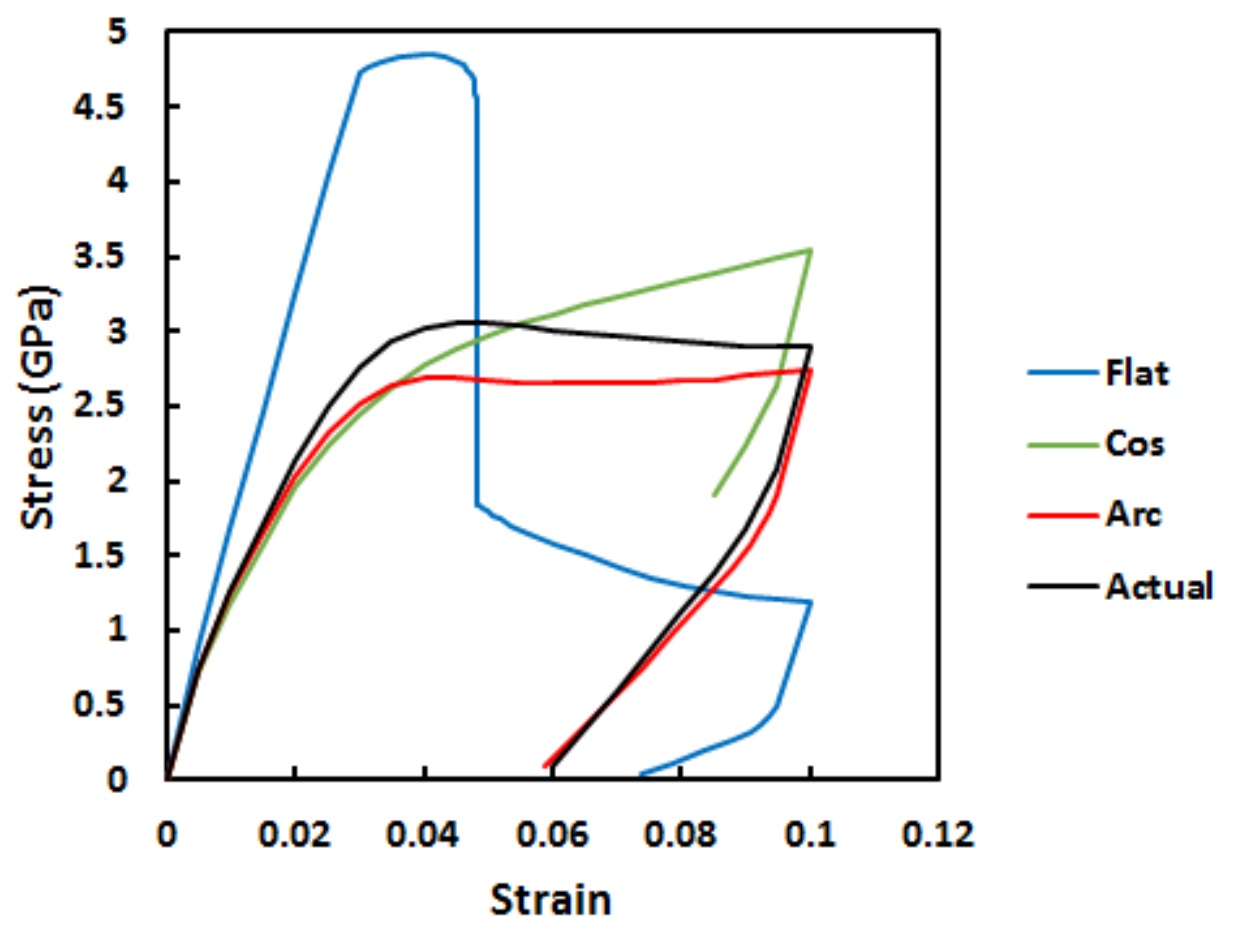

28 

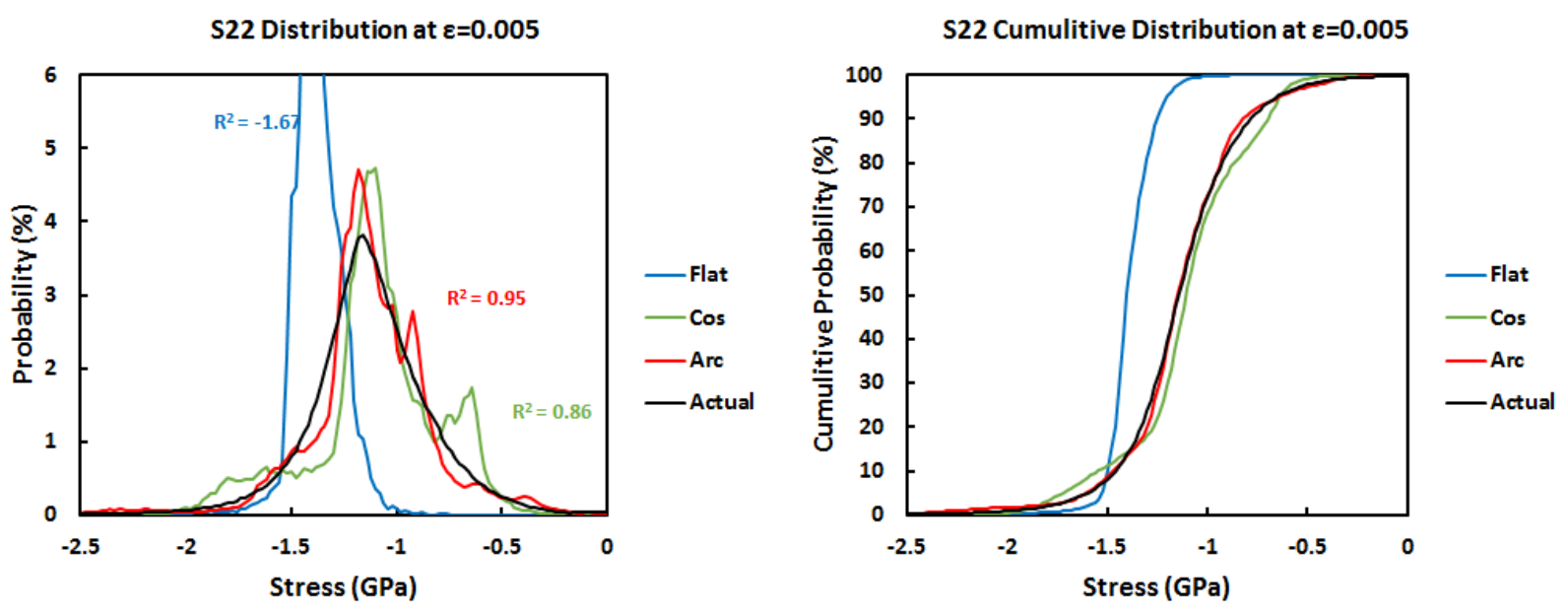